\title{
Dynamic Analysis of Superconductor at High Temperature for Antennas Array
}

\author{
H. C. C. Fernandes, L. M. Caetano and H. M. C. A. Maia \\ Department of Electrical Engineering, Federal University of Rio Grande do Norte \\ P. O. Box 1583, Natal/RN 59.078-970, Brazil \\ humbeccf@ct.ufrn.br, leoteleco@yahoo.com.br and humychel@yahoo.com.br
}

\begin{abstract}
The analysis and new results of the resonance frequency and radiation pattern of antennas array, for glass fiber FR-4 substrate using superconductor and copper patch, are presented. The linear superconducting rectangular antennas array uses the new materials SnBaCaCuOy at temperature of $160 \mathrm{~K}$ with conductivity equal $1.5 \times 10^{5} \mathrm{~S} / \mathrm{m}$. The concise full wave Transverse Transmission Line (TTL) method is used in the analysis and to obtain some results. The HFSS is also used and new results for the reflection coefficient $\left(S_{11}\right)$, return loss as functions of the resonant frequency, and pattern radiation of antennas array, are presented.
\end{abstract}

Index Terms - antennas array, superconductor, terahertz, TTL method.

\section{INTRODUCTION}

The discovery of new superconducting materials has attracted an enormous interest in the scientific community world, because of its technological potential in terms of devices applications as antennas, sensors, detectors. A superconducting material shows two properties: very low (almost zero) resistance passage of electric current and perfect diamagnetism.

In a normal conductor, an electrical current decreases rapidly due to the resistance of the material. For the superconducting materials the electrical current without resistance, the power is not lost when it flows through of them. The superconductivity is a state of matter observed only in some solids, mostly metals.

Regarding the study of superconductivity, there is a phenomenon that was discovered in 1933 by researchers H. Meissner and R. Ochsenfeld, which reports the perfect diamagnetism, which means that lines of magnetic flux are completely expelled from the superconductor and there is a force that repels the superconducting away from magnetic fields. This phenomenon became known as the Meissner effect [1-3].

A theory widely used for superconductor it is the BCS theory, developed by Bardeen, Cooper and Schrieffer. 
The macroscopic theory uses the two fluids model and the London equations. In this work are used antennas arrays with superconductor patch, according with structure shown in Fig. 1, only for a radiating element.

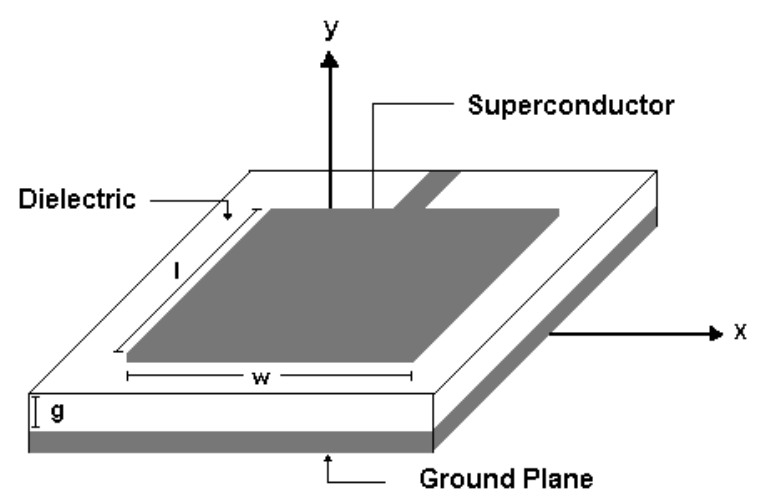

Fig. 1. Microstrip antenna with superconducting patch of width, $w$, and length, $l$.

Considering the microstrip antenna resonator of Fig. 1, the equations that represent the electromagnetic fields in the $\mathrm{x}$ and $\mathrm{z}$ directions as function of the electric and magnetic fields in the $\mathrm{y}$ direction are obtained, applying the TTL method [1]-[4]. The concise full wave Transverse Transmission Line (TTL) method is used in the analysis and to obtain new results in GHz and Terahertz frequency. The HFSS is also used and new results for the reflection coefficient $\left(S_{11}\right)$, return loss as functions of the resonant frequency, and pattern radiation of antennas array, in 2D and 3D, are presented.

The linear superconducting rectangular antennas array uses the new materials $\mathrm{SnBaCaCuOy}$ at temperature of $160 \mathrm{~K}$ with conductivity equal $1.5 \times 10^{5} \mathrm{~S} / \mathrm{m}$. The superconducting material is the characterization of chemical compounds and laboratory tests, where the analysis of the $160 \mathrm{~K}$ compound, dubbed Sn-3427.

\section{THEORY}

Starting from the Maxwell's equations, after various algebraic manipulations, the general equations for the antenna in the FTD - Fourier Transformed Domain are obtained, for the $\mathrm{x}$ and $\mathrm{z}$ field components directions as [1] , [5]. The proposed structure is analyzed by the Transverse Transmission Line - LTT method, which uses the "y" direction transverse to the dielectric interfaces, with $E_{y}$ and $H_{y}$ components. Then, separating the fields in " $x$ " and " $z$ " directions, of the terms in the " $y$ " direction, the general equations for the electromagnetic fields to the device under study, in an arbitrary region are given by: 


$$
\begin{aligned}
& \tilde{E}_{x i}=\frac{1}{\gamma_{i}^{2}+k_{i}^{2}}\left[-j \alpha_{n} \frac{\partial}{\partial y} \tilde{E}_{y i}+\omega \mu \beta_{k} \tilde{H}_{y i}\right] \\
& \tilde{H}_{x i}=\frac{1}{\gamma_{i}^{2}+k_{i}^{2}}\left[-j \alpha_{n} \frac{\partial}{\partial y} \tilde{H}_{y i}-\omega \beta_{k} \tilde{E}_{y i}\right] \\
& \tilde{E}_{z i}=\frac{1}{\gamma_{i}^{2}+k_{i}^{2}}\left[-j \beta_{k} \frac{\partial}{\partial y} \tilde{E}_{y i}-\omega \mu \alpha_{n} \tilde{H}_{y i}\right] \\
& \tilde{H}_{z i}=\frac{1}{\gamma_{i}^{2}+k_{i}^{2}}\left[-j \beta_{k} \frac{\partial}{\partial y} \tilde{H}_{y i}+\omega s \alpha_{n} \widetilde{E}_{y i}\right]
\end{aligned}
$$

Where:

$\mathrm{i}=1,2$ are the dielectric regions of structure;

$\gamma_{\mathrm{i}}{ }^{2}=\alpha_{\mathrm{n}}{ }^{2}+{\beta_{\mathrm{k}}}^{2}-\mathrm{k}_{\mathrm{i}}^{2}$ Is the propagation constant in $\mathbf{y}$ direction;

$\alpha_{n}$ is spectral variable in $\mathbf{x}$ direction;

$\beta_{\mathrm{k}}$ is spectral variable in $\mathbf{z}$ direction;

$\mathrm{k}_{\mathrm{i}}{ }^{2}=\omega^{2} \mu \varepsilon=\mathrm{k}_{0}{ }^{2} \varepsilon_{\mathrm{ri}}^{*}$ is the wave number of the dielectric region;

$\varepsilon_{\mathrm{ri}}^{*}=\varepsilon_{\mathrm{ri}}-\mathrm{j} \frac{\sigma_{\mathrm{i}}}{\omega \varepsilon_{0}}$, is the relative dielectric permittivity of the complex material.

To obtain the unknown constants of magnetic and electric fields were applied the boundary conditions, at the interface between the dielectric regions. Also the dyadic Green $\mathrm{Z}$ functions is obtained using the magnetic boundary conditions,

$$
\left[\begin{array}{c|c}
Z_{x x}-Z_{S} & Z_{x z} \\
---- & ---- \\
Z_{z x} & Z_{z z}-Z_{S}
\end{array}\right] \cdot\left[\begin{array}{c}
\tilde{J}_{x} \\
--- \\
\tilde{J}_{z}
\end{array}\right]=\left[\begin{array}{l}
\tilde{E}_{x}^{\text {out }} \\
-- \\
\tilde{E}_{z}^{\text {out }}
\end{array}\right]
$$

Where:

$$
Z_{S}=\frac{1}{\sigma_{S} t}
$$

$\mathrm{Zs}$ is the superconductor impedance, $\sigma_{s}$ is the conductivity and " $t$ " is the thickness of the superconductor patch.

The Moment method is used to eliminate the electric fields in (5), and to obtain the homogeneous matrix equation (7) for the calculation of the complex resonant frequency. 


$$
\left[\begin{array}{ll}
K_{x x} & K_{x z} \\
K_{z x} & K_{z z}
\end{array}\right] \cdot\left[\begin{array}{l}
a_{x} \\
a_{z}
\end{array}\right]=\left[\begin{array}{l}
0 \\
0
\end{array}\right]
$$

The determinant of this $\mathrm{K}$ matrix, witch is the inner product of the $\mathrm{Z}$ matrix and the basis functions, provides the real and imaginary resonant frequencies, which are numerically calculated using computational programs developed by the authors, in Fortran Power Station Language .

One of the problems when working with photonic material, is the relative dielectric constant determination as the PBG, is a non-homogeneous structure where the incident sign goes at the process of multiple spread. A solution can be obtained through a numerical process called homogenization [8]. The process is based in the theory related to the diffraction of an incident electromagnetic plane wave, imposed by the presence of an air immerged cylinders in a homogeneous material. For electromagnetic waves propagating in the xy plane this waves have the s polarization (E field parallel to the $\mathrm{z}$ axis) and $p$ polarization (E field perpendicular to the $\mathrm{z}$ axis).

\section{SUPERCONDUCTOR}

The quantum theory of the superconductivity [6], [11] was launched in 1957 by the work of Bardeen, Cooper and Schieffer.

An attractive interaction between electrons can be conduced to a ground plane, separated from an excited state by an energy gap, which separates the superconducting electrons below of the gap of the normal electrons. The critical field, the thermal and electromagnetic properties are many other consequences of this energy gap. All elements of the array are fed each other. A signal is generated via network analyzer and this is inserted in the microstrip line coaxial cable $50 \Omega$ impedance and thus this signal propagates to other elements of the arrangement for microstrip lines of same size of the previous.

The penetration depth, $\lambda$ appear as natural consequences of the BCS theory [3], [10]. The London equation is obtained at the magnetic fields, which vary slowly in space. Thus the Meissner effect is obtained naturally.

The BCS theory predicts the critical temperature of an element or alloy. There is a paradox: the greater resistivity at room temperature is the likelihood that this metal is a superconductor when cooled.

The London equations are used [10],

$$
e \vec{E}=m \frac{d \vec{v}}{d t}
$$




$$
\Lambda \frac{\partial \vec{j}}{\partial}=\vec{E}
$$

Where, $\Lambda$ is London constant, $\mathrm{m}$ is the electrons number, $e$ is the electron charge and $\mathrm{v}$ is the Fermi velocity.

\section{ANTENNAS ARRAY}

The antennas array [4] consists of a finite number of identical irradiant elements, which combines the induced signals in these antennas, to form the array. The maximum beam direction is controlled, adjusting the phase of the sign in different elements. The phase induced in the several adjustments in the elements, permits to obtain maximum directivity and gain.

The antennas array can be classified as linear and planar. The linear array factor is,

Where:

$$
F A=\frac{\sin (N \psi)}{\sin (\psi / 2)}
$$

$$
\psi=k d \cos \theta+\beta
$$

The Fig. 2 shows the linear array with superconductor patch antenna with three elements. This array, is suitable for simulation by commercial software HFSS, which is based on the finite element method.

In this work the Transverse Transmission Line (TTL) method is also applied, using double Fourier Transform [10-12], and considering EBG, Terahertz frequency.

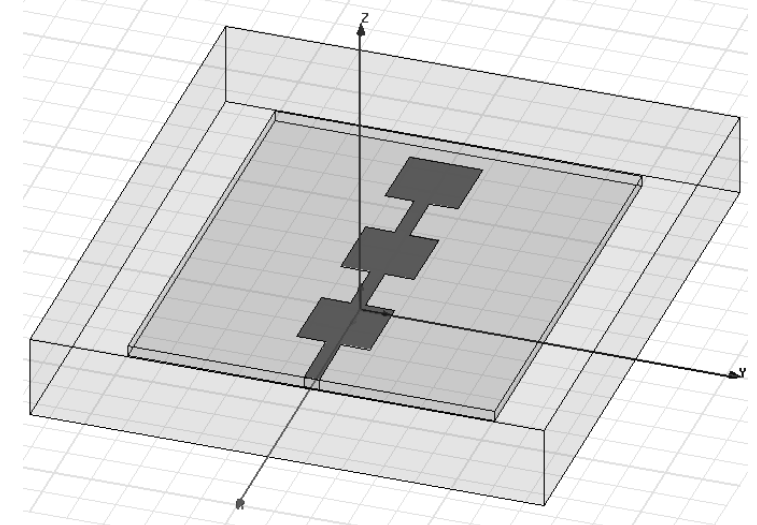

Fig. 2. Linear antennas array with superconducting patch using HFSS.

\section{RESULTS}

The Fig. 3 shows the comparative results for return loss as function of the resonant frequency, for FR-4 substrates using superconductor and copper patches. The thickness of the superconductor patch 
utilized in this work is $0.05 \mathrm{~mm}$, in Figs. 3 until 8. This value considered is the same for the two cases and has the same value the perfect electric conductor.

The black curve shown the results using FR-4 substrate with $\varepsilon_{\mathrm{r}}=4.4$, length $=60.0 \mathrm{~mm}$, width $=$ $50.0 \mathrm{~mm}$, substrate thickness $=1.48 \mathrm{~mm}$ with superconductor patch $\mathrm{SnBaCaCuOy}$, while the red curve shown the results using copper patch, with the same FR-4 substrate.

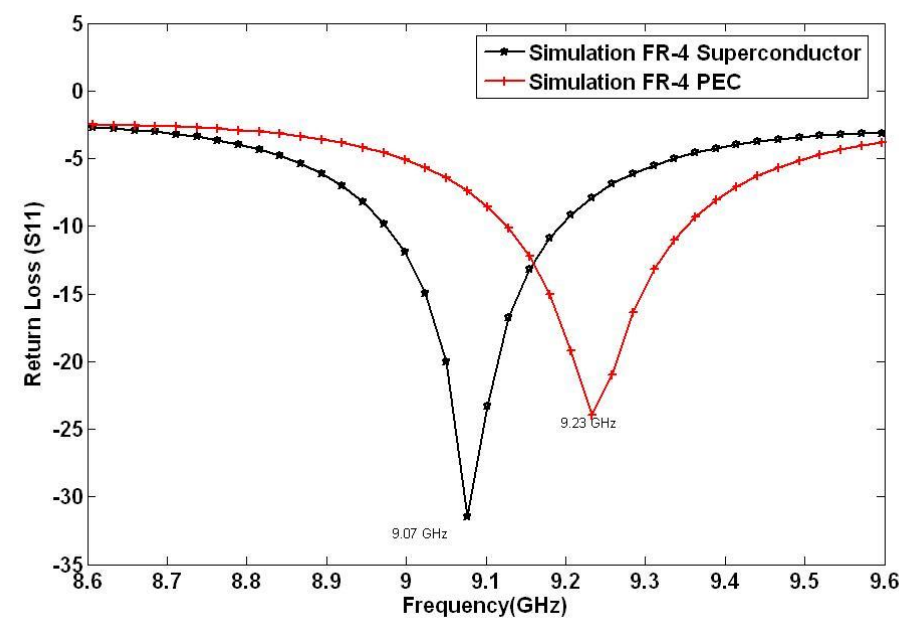

Fig. 3. Comparatives curves of return loss versus frequency in HFSS for FR-4 substrate with superconducting material and copper patches.

According with the Fig. 3, the superconducting patch presents resonances more efficient using the FR-4 as substrate, where the ressonance frequency is $9.07 \mathrm{GHz}$ with a reflection coefficient (S11) of $31 \mathrm{~dB}$, while the use of the copper patch presents $9.23 \mathrm{GHz}$ with a reflection coefficient (S11) of -25 $\mathrm{dB}$. The superconductor patch showed a bandwidth of approximately $250 \mathrm{MHz}$. In figure 3 we can to observe a band of frequencies varying between $8.6 \mathrm{GHz}$ to $9.6 \mathrm{GHz}$, characterized by being frequencies of the band X. It was observed that the resonant frequency of the copper patch had a reduction of $1.73 \%$ compared to the resonance frequency of the superconductor patch, equivalent to a decrement in the $160 \mathrm{MHz}$ operating frequency. This also results in a decrease in the $250 \mathrm{MHz}$ bandwidth.

Fig. 4 shows the comparative results for return loss as a function of resonance frequency of FR-4 substrates, using superconductor for two and three elements of the antenna array, at $160 \mathrm{~K}$. The black curve shows the results using two elements, FR-4 substrate with $\varepsilon_{\mathrm{r}}=4.4$ and $\mathrm{h}=1.54 \mathrm{~mm}$, length $=10$ $\mathrm{mm}$, width $=10 \mathrm{~mm}, \mathrm{SnBaCaCuOy}$ superconducting patch, while the red curve shown in the results using three elements, with FR-4 substrate and same materials. 


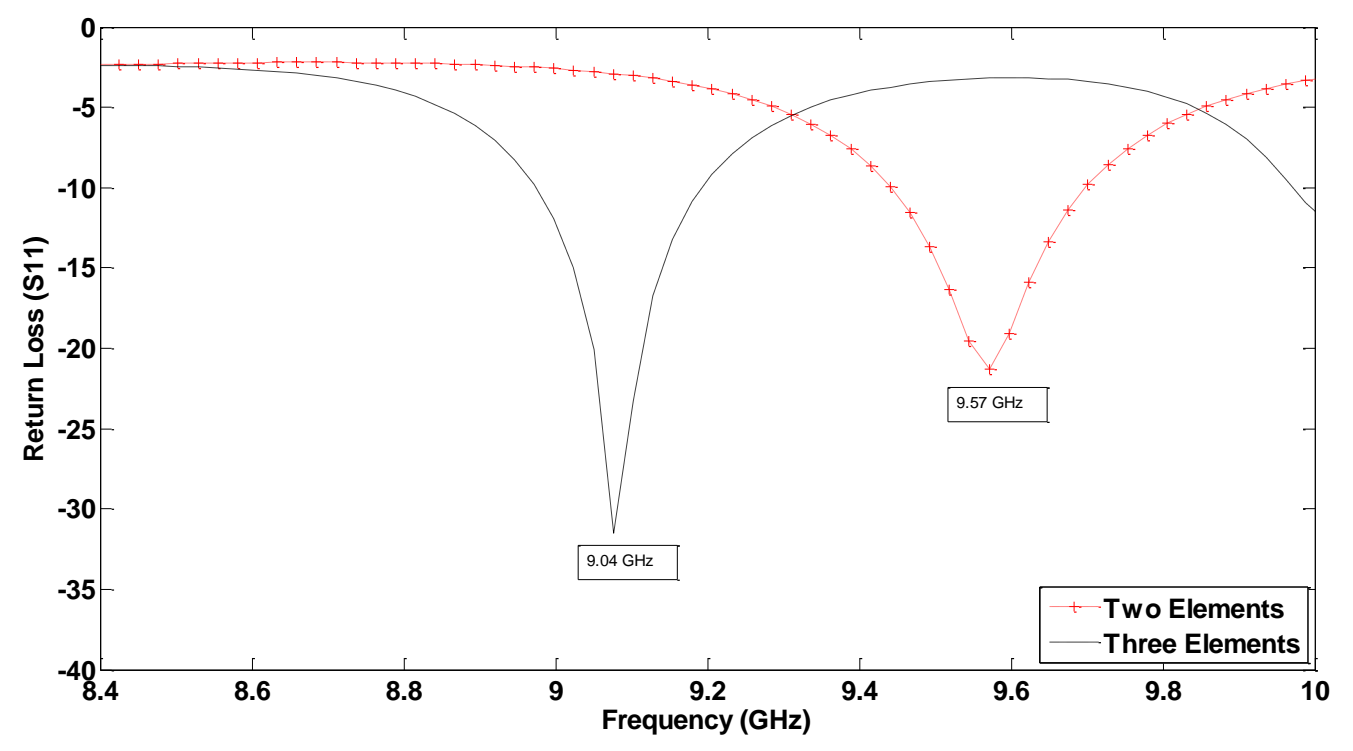

Fig. 4. Return loss versus frequency using HFSS for FR-4 substrate, $\varepsilon_{\mathrm{r}}=4.4$ with superconducting materials of linear arrays, with two and three elements, at $160 \mathrm{~K}$.

The resonances using two elements superconductive, is $9.57 \mathrm{GHz}$ with a reflection coefficient (S11) of $-22 \mathrm{~dB}$, while the use of three elements presents resonance of $9.04 \mathrm{GHz}$, with a reflection coefficient (S11) of $-32 \mathrm{~dB}$.

The radiation patterns two dimensional array of these antennas shown in Fig. 5 and 6 have different gain values according to the resonance frequency. The red curve represents the total gain of the antenna in E-Plane and H-Plane. While the purple curve representing the overall gain of E-Plane and H-plane, using an FR-4 substrate, with the superconducting material to a linear array of two elements (Fig. 5), and linear array of three elements (Fig. 6), at 160K. In this case, it can be observed that the linear array of three elements is more directive, than that array of two elements. The validation of these theoretical results is made through comparison with published work in the same line of research, such as the work cited in [13] that shows prospects radiation pattern and resonant frequency. 


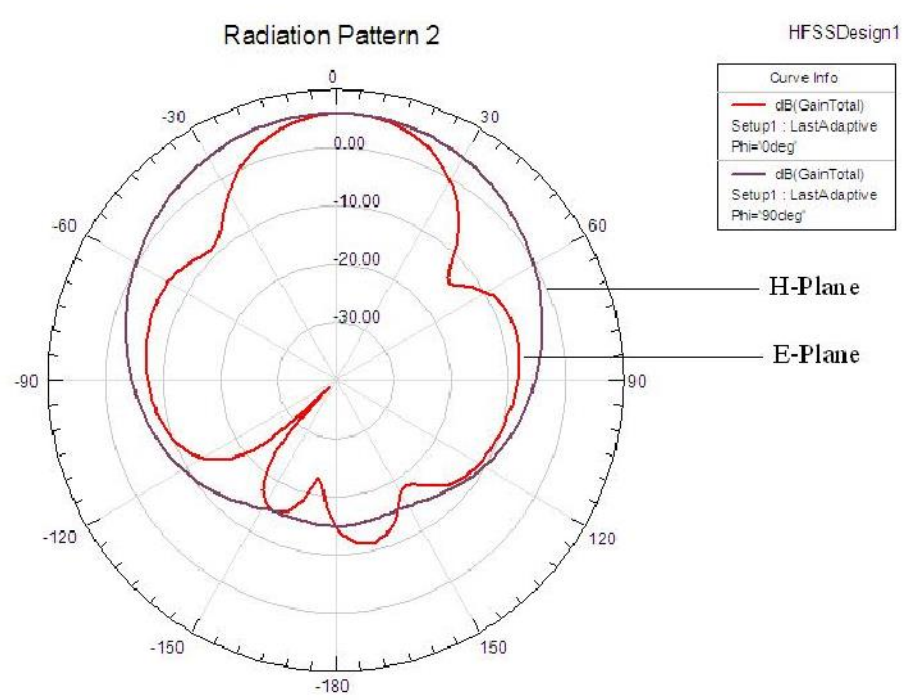

Fig. 5. 2-D radiation pattern of antenna array with two elements, using FR-4 substrate, with superconductor material, at $160 \mathrm{~K}$.

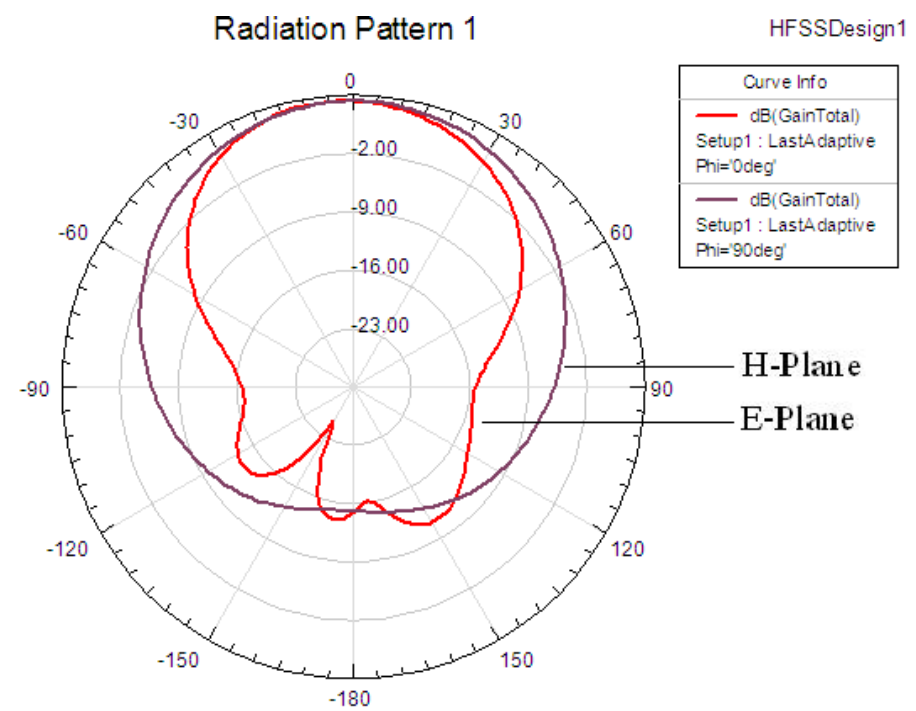

Fig.6. 2-D radiation pattern of antenna array with three elements, using FR-4 substrate, with superconductor material, at $160 \mathrm{~K}$.

Figures 7 and 8 shows the three-dimensional radiation patterns of the array antennas simulated in HFSS, with superconductor patches, using FR-4 substrate with two and three radiating elements, respectively.

According to the simulation gain peak for the patch with FR-4 substrate using patch with three superconducting elements is approximately $5.96 \mathrm{~dB}$, and gain array of two elements with FR-4 substrate is $4.32 \mathrm{~dB}$. 


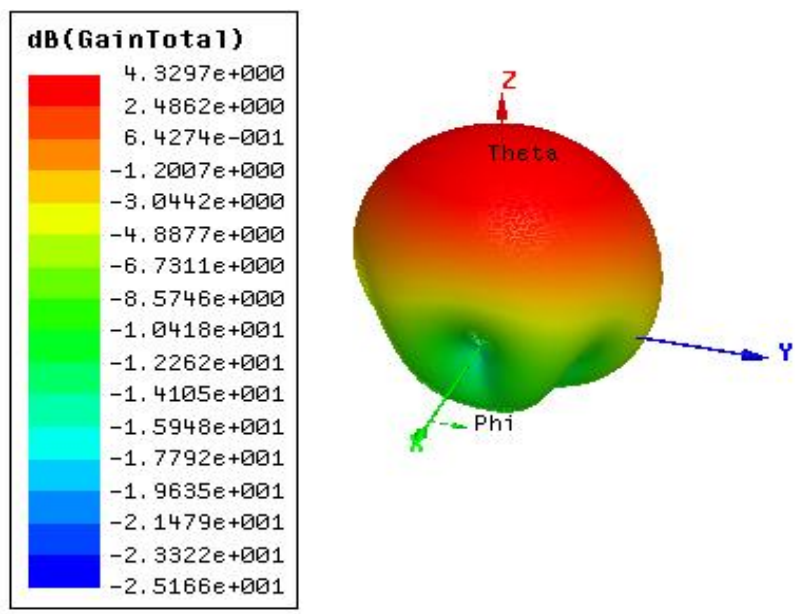

Fig. 7. 3-D radiation pattern of antenna array with two elements using FR-4 substrate with superconductor material at $160 \mathrm{~K}$.
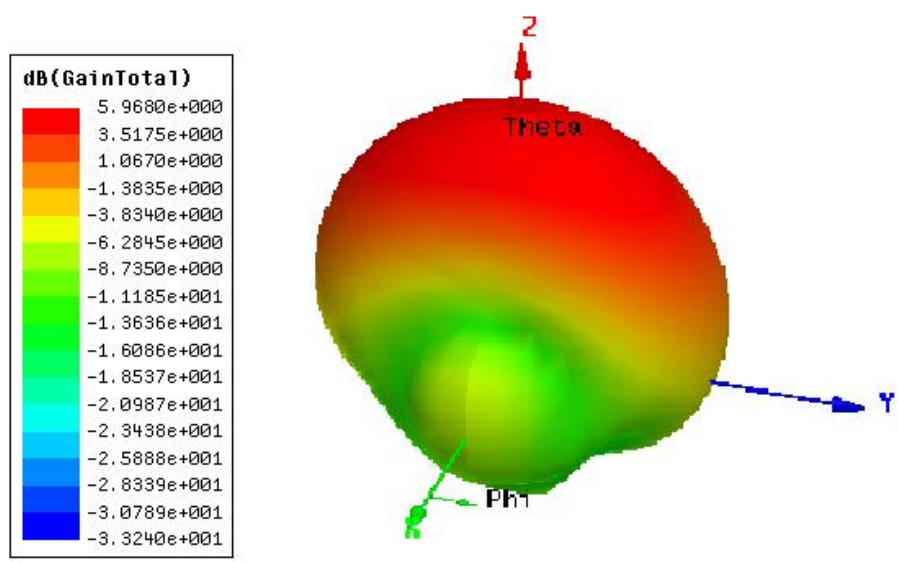

Fig. 8. 3-D radiation pattern of antennas array with three elements, for FR-4 substrate with superconductor material at $160 \mathrm{~K}$.

The Fig.9 shows the resonance frequency in $\mathrm{GHz}$ as function of the patch length, for $90 \mathrm{~K}$ and $160 \mathrm{~K}$ critical temperatures, using the TTL method, using computational programs developed and FORTRAN power Station and Matlab. The parameters used are, $\mathrm{g}=0.0154 \mathrm{~mm}, \mathrm{w}=0.03 \mathrm{~mm}$ and substrate $\varepsilon_{\mathrm{r} 1}=$ 8.729 , at $90 \mathrm{~K}$ and $160 \mathrm{~K}$, thickness of the patch, $\mathrm{t}=10 \mathrm{~mm}$. 


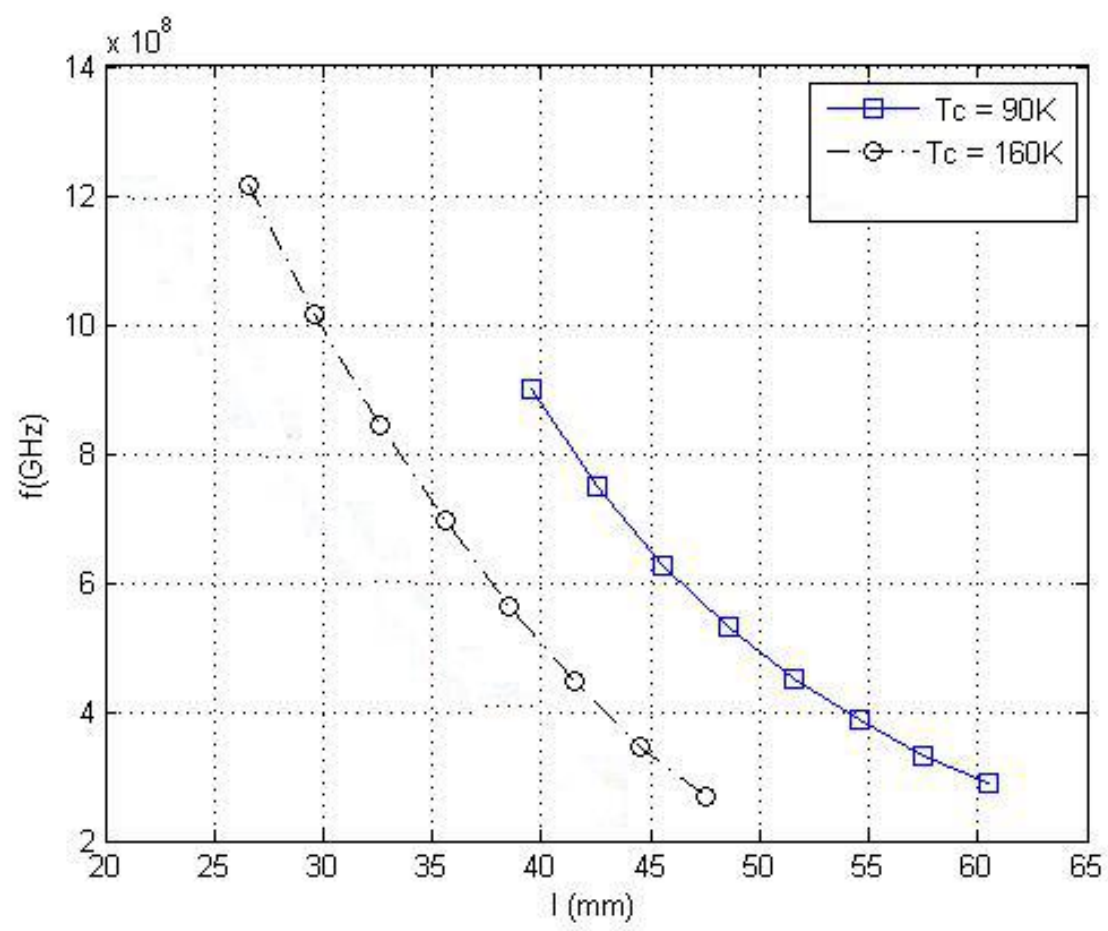

Fig.9. Resonance frequency in $\mathrm{GHz}$ as functions of the patch length, at critical temperature of $90 \mathrm{~K}$ and $160 \mathrm{~K}$ and substrate $\varepsilon_{\mathrm{r} 1}=8.729$.

Fig. 10 shows the resonance frequency and EBG (Electromagnetics Band Gap) substrate, the red curve shows the results using two elements, with $\varepsilon_{\mathrm{r}}=10.233$ and $\mathrm{h}=1.54 \mathrm{~mm}$, width $=5 \mathrm{~mm}$, while the blue curve shown in the results using $\varepsilon_{\mathrm{r}}=8.729$ and $\mathrm{h}=1.54 \mathrm{~mm}$, width $=5 \mathrm{~mm}$, both curves with superconductor patch at $150 \mathrm{~K}$ with critical temperature at $160 \mathrm{~K}$, thickness of the patch, $\mathrm{t}=10 \mathrm{~mm}$.

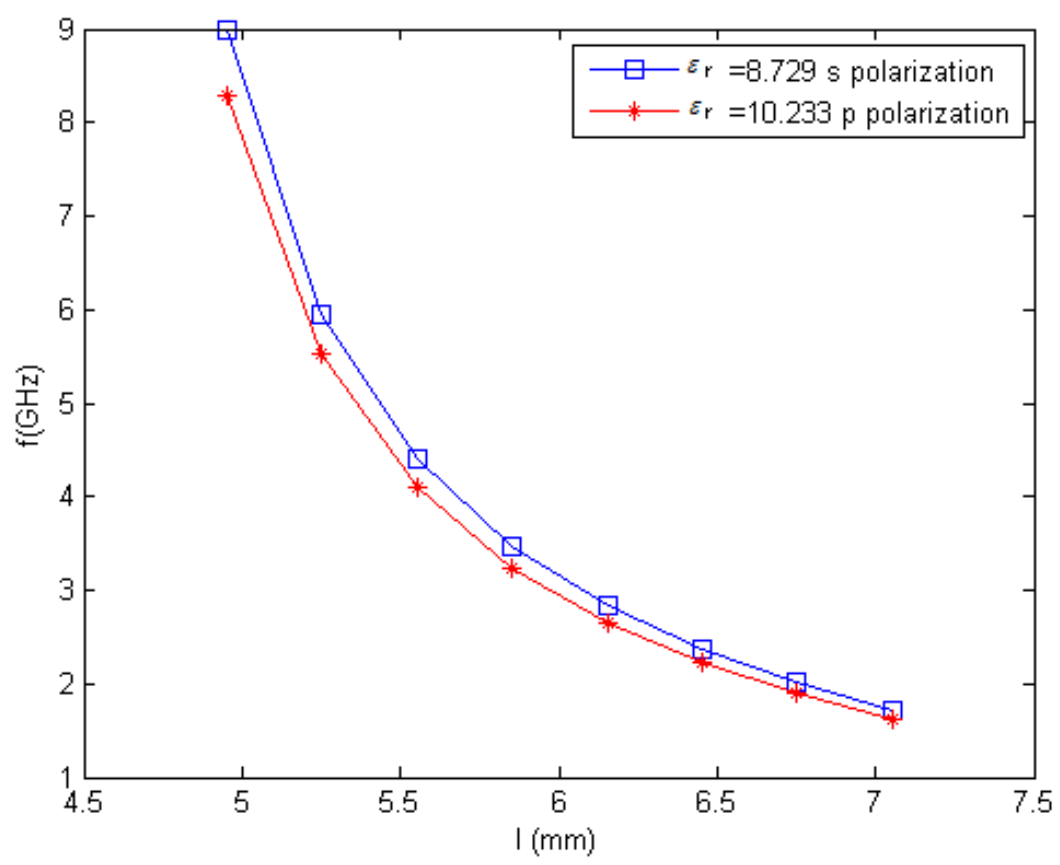

Fig. 10. Resonance frequency as functions of the length, using TTL method. 
The Fig.11 shows the resonance frequency in $\mathrm{THz}$ as function of the patch length, for $160 \mathrm{~K}$ critical temperature, using the TTL method. The parameters used are, $\mathrm{g}=0.0154 \mathrm{~mm}, \mathrm{w}=0.03 \mathrm{~mm}$ and $\varepsilon_{\mathrm{r} 1}$ $=4.4$, thickness of the patch, $\mathrm{t}=0.1 \mathrm{~mm}$.

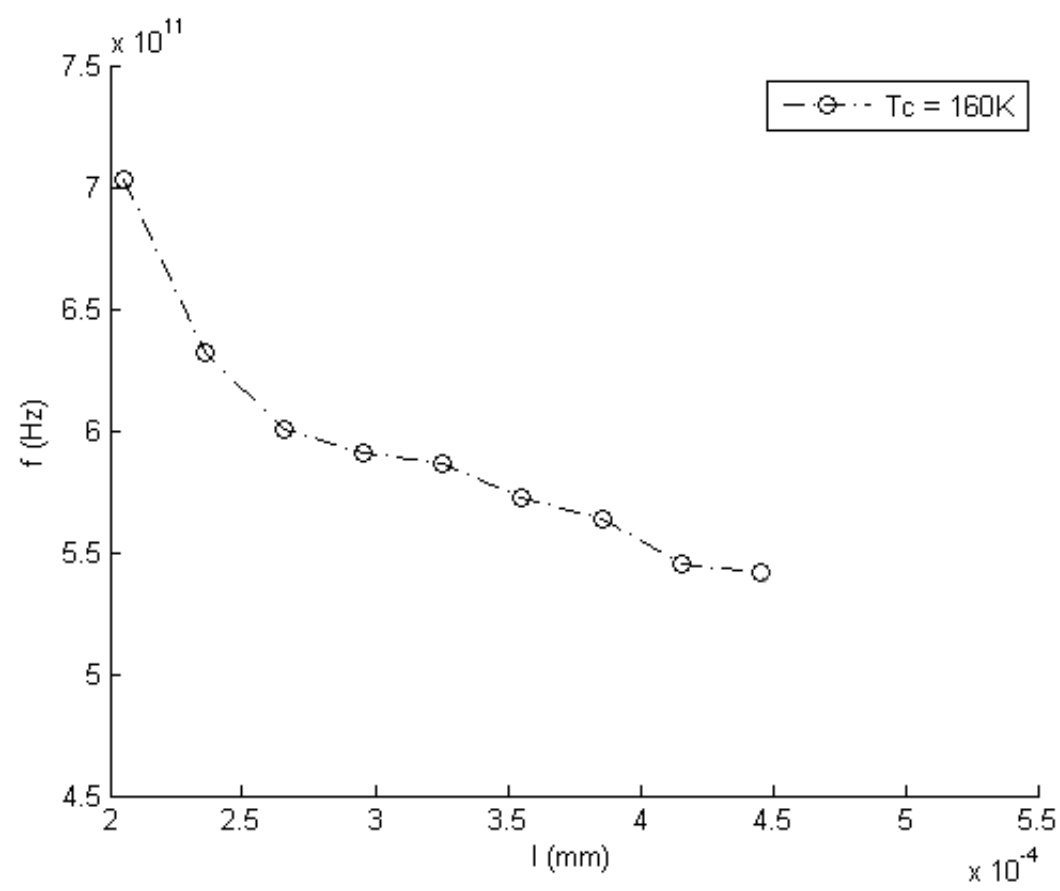

Fig.11. Resonance frequency in $\mathrm{THz}$ as functions of the patch length, at critical temperature of $160 \mathrm{~K}$ and substrate $\varepsilon_{\mathrm{r} 1}=4.4$.

\section{CONCLUSION}

In this paper, linear antennas array with two and three elements with superconductor patch was simulated by the HFSS, using different substrate with comparative results using superconductor and copper patches. The numerical results showed that the linear antennas array, using superconductor material, presents better performance than that of copper, providing better radiation efficiency. The Transverse Transmission Line (TTL) method was also used and new results of the resonant frequency, in Terahertz were presented. Values of higher critical temperature of $160 \mathrm{~K}$ were used.

The superconducting theories have been presented at new superconductor materials. Numerical results of the resonance frequency as functions of the length were presented, including two dimensional and three dimensional radiation pattern of the E-plane and H-plane.

It is known that the impedance elements can be drastically affected by the excitation of surface waves on the device. In this work, the mathematical modeling is analyzed to the patch with an element. It is subsequently performed an extension to three elements, in order to analyze the effect that occurs in terms of resonant frequency, return loss and bandwidth, as well as this contributes to the radiation characteristics in far fields.

\section{ACKNOWLEDGMENT}

The authors wish to acknowledge the assistance and support of CNPq and CAPES, Brazilian agencies. 


\section{REFERENCES}

[1] L. M. Caetano, H. C. C. Fernandes and H. M. C. A. Maia, "Analysis of Planar Structure with Patch Superconductor Material and PBG Substrate". In: 3rd Internacional Conference on Metamaterials, Photonic Crystals and Plasmonics, 2012, Paris. META12, Apr. 2012.

[2] L. M. Caetano, H. C. C. Fernandes and H. M. C. A. Maia, "Microstrip Antennas Array with superconductor patch at High Temperature at 254K.”, In: International Conference on Superconductivity and Magnetism, 2012, Istanbul, Apr. 2012.

[3] H. M. C. A. Maia, L. M. Caetano, H. C. C. Fernandes, "Rectangular Microstrip Antennas Linear Array with Superconductor at High Temperature”, In: World Congress in Computer Science Computer Engineering and Applied Computing, 2011, Las Vegas. ICWN 2011 International Conference on Wireless Networks, 2011.

[4] Oliveira, E. E. C.; Da Assunção, A. G; Oliveira, E. E. C.; Oliveira, J. B. L.; Cabral, A. M. Small size quasi-fractal microstrip antenna M1 EBG-GP. International Journal of Applied Electromagnetics and Mechanics, V. 39, p. 645$649,2012$.

[5] H. C. C. Fernandes, R. R. C. Franca and D.B. Brito, "Asymmetric Fin Line and Coupler at Millimeter Waves on PBG Substrate”, Journal of Infrared, Millimeter, and Terahertz Waves, V. 32, No 1, pp. 116-125, Jan. 2011.

[6] E. B. Eckholm and S. W. Mcknight, “Attenuation and Dispersion for High-Tc Superconducting Microstrip Lines”, IEEE-MTT, Vol. 38, pp. 387-395, 1990.

[7] D. Nghiem, J. T. Williams and D. R. Jackson, "A General Analysis of Propagation Along Multiple-layer Superconducting Stripline and Microstrip Transmission Lines”, IEEE-MTT, Vol. 39, No 9, pp. 1553-1565, Set.1991.

[8] E. Centeno and D. Felbacq, "Rigorous vector diffraction of electromagnetic waves by bidimensional photonic crystals”, J. Optical Soc. American A/Vol. 17, No.2, pp.320-327, Feb. 2000.

[9] Z. Cai and J. Bornemann, "Generalized Spectral-Domain Analysis for Multilayered Complex Media and High-Tc Superconductor Application”, IEEE-MTT, Vol. 40, No 12, pp. 2251-2257, Dez. 1992.

[10] Zhi-Yuan Shen, "High-Temperature Superconducting Microwave Circuits”, Artech House, Inc. 1994.

[11] H. C. C. Fernandes, J. R. S. Oliveira and A. J. Giarola, "Dispersion in Finlines on Semiconductor and the SParameters of a Step Discontinuity”, International Journal of Infrared and Millimeter Waves, Vol. 12, No 5, pp. 505519, USA, May, 1991.

[12] H. C. C. Fernandes and H. M. C. A. Maia, "New Antenna with Superconductor at Critical Temperature of 212". IMOC2009- SBMO I EEE MTT-S International Microwave and Optoelectronics Conference, Belem - PA, Brazil, paper 59111. pp. 197-199,Nov. 2009.

[13] L. L. Lewis and G. Koepf, "Performance of TiCaBaCuO $30 \mathrm{GHz} 64$ elements antenna array" IEEE Transactions on applied superconductivity, VOL 3 No.1 Mar. 1993. 